\title{
HPV detection methods
}

\author{
Antoinette A.T.P. Brink, Peter J.F. Snijders and Chris J.L.M. Meijer* \\ VU University medical center, Department of Pathology, Section Molecular Pathology, De Boelelaan 1117, 1081 \\ HV Amsterdam, The Netherlands
}

\begin{abstract}
Given the causal relation between a persistent high-risk human papillomavirus (hrHPV) infection and the development of high-grade cervical intraepithelial neoplasia (CIN) and cervical cancer, hrHPV testing has been advocated in addition to cytology for the detection of clinically relevant cervical lesions. HrHPV testing is thought to improve cervical screening algorithms, the management of women with cytologically equivocal smears, and the management of women treated for high grade CIN. In this chapter we discuss different methods for HPV detection and genotyping and their respective applications.
\end{abstract}

Keywords: Human papillomavirus, polymerase chain reaction, reverse hybridization, hybrid capture, real-time PCR, cervical carcinoma, NASBA, DNA probes, epidemiology

\section{Introduction}

Cervical cancer is preceded by premalignant lesions termed cervical intraepithelial neoplasia (CIN). Cervical cancer screening largely relies on cytological detection of these premalignant lesions, which can be treated relatively easily and with minor side effects. Given the causal relation between a persistent high-risk human papillomavirus (hrHPV) infection and the development of high-grade CIN and cervical cancer, hrHPV testing has been advocated in addition to cytology for the detection of clinically relevant cervical lesions. HrHPV testing is thought to improve cervical screening algorithms [1], the management of women with cytologically equivocal smears [2,3], and the management of women treated for high grade CIN [4]. In this chapter we elaborate on the different methods for HPV detection and their respective applications.

\subsection{HPV phylogeny}

HPVs are associated with various benign and malignant epithelial proliferative diseases. Over 100 geno-

* Corresponding author: Prof. Dr. C.J.L.M. Meijer, Dept. Pathology, VU University medical center, PO Box 7057, 1007MB Amsterdam, The Netherlands. Tel.: +31 20 4444070; Fax: +31 20 4442964; E-mail: cjlm.meijer@vumc.nl. types of HPV are recognized. An HPV isolate is described as a novel type if the nucleotide sequence of its E6, E7 and L1 genes differs more than $10 \%$ from that of any other HPV type. Based on tropism, a distinction can be made between cutaneous HPV types that infect the epidermis, and mucosal HPV types that infect the epithelia of the anogenital or the aerodigestive tract.

In vitro studies showed that a group of phylogenetically related mucosal HPV types have oncogenic potential: their E6 and E7 proteins interfere with cell cycle regulation by mediating degradation of $\mathrm{p} 53$ and $\mathrm{pRb}$ proteins, respectively [5,6]. Exactly these HPV types are an important causal factor in carcinogenesis of the uterine cervix and are therefore designated "high-risk" (hr) [7]. Epidemiological studies have shown that these hrHPVs include types 16, 18, 31, 33, 35, 39, 45, 51, 52, $56,58,59,68,73$ and 82 , and probably also types 26 , 53 and 66 [8]. In the phylogenetic tree of HPV types, hrHPVs cluster in the groups designated A5, A6, A7, A9 and A11 [9]. Non-oncogenic or low-risk genital HPVs (lrHPVs) are phylogenetically distant from the hrHPVs. They include HPV 6, 11, 40, 42, 43, 44, 54, 61, 70, 72, 81, and CP6108. Their E6 and E7 proteins are less competent in interfering with p53 [10] and $\mathrm{pRb}$ [11] functions than the E6 and E7 proteins of hrHPVs. LrHPVs can cause benign proliferations such as condylomata acuminata (genital warts). For a number of mucosal HPV types, the risk has not been 
determined yet [8]. However, some of them could be considered as potentially oncogenic due to their phylogenetic relationship with known hrHPV types. For example HPV type 67, which initially was isolated from a vaginal intraepithelial neoplasia (VIN) [12], clusters in the A9 group that contains only hrHPVs such as HPV16 [9].

\subsection{The need for HPV genotyping}

Many HPV tests rely on cocktails of probes representing 13 or 14 of the most common hrHPV types. It has been suggested that the added value of less frequent hrHPV types to such probe cocktails would be small, probably irrelevant for screening programs, and resulting in a substantial decrease in screening specificity $[13,14]$. However, the increasing need to distinguish individual hrHPV types is illustrated by several current developments in the field of HPV research. Firstly, there is growing evidence that certain hrHPV types confer increased risks for high-grade CIN and cervical carcinomas. This is discussed in more detail in the next paragraph. Secondly, 20-30\% of HPV infections involve multiple HPV types and in those cases typing is necessary to determine the contribution of the individual HPV types. Thirdly, there is an increasing interest in prophylactic HPV vaccination (reviewed in [15]), which entails studies of geographical distribution of HPV types and their associated diseases as a basis for prophylactic vaccination programs. Once vaccination has started, HPV typing remains necessary to monitor the changes in prevalence of the type(s) represented in the vaccines as a measure for vaccine efficacy.

HPV genotyping may also have some advantages and perhaps some prognostic value in monitoring of women who have been treated for CIN3 or cervical cancer, although the treatment itself does not depend on the typing result. Demonstrating the same hrHPV type in the post-treatment specimen as in the primary CIN3 lesion may indicate a recurrent hrHPV infection due to incomplete removal of the lesion or disability of the respective woman to clear a particular hrHPV type $[16,17]$ and might require more intense follow-up or more aggressive treatment.

Finally, the number of known HPV types is still increasing. Although novel HPV types are often isolated from malignancies, their oncogenic potential can only be deduced from a combination of in vitro and epidemiological studies, the latter of which should include typing.

\subsection{HrHPV types and risk of high-grade cervical lesions}

We showed that in the Dutch screened population [18], HPV types 16 and 33 are more prevalent in women with a cytological reading of moderate dyskaryosis or worse and underlying CIN2 or worse, than in women with normal cytology. This suggests that infection with these types confers an increased risk for development of high-grade cervical lesions [19].

Moreover, HPV type distribution in women with cervical carcinoma as compared with cytologically normal women showed that HPV18 is mainly a risk factor for the development of adenocarcinoma (AdCa) and its precursor adenocarcinoma in situ (ACIS), whereas HPV16 is associated with both squamous cell carcinoma (SCC) and AdCa [20]. In line with the abovementioned findings, an increased risk posed by HPV16 and HPV18 for cervical (pre)cancers of the squamous and adeno-histotypes was shown in a prospective screening cohort of 20810 women followed for up to 10 years [21]. In addition, an increased risk for cervical precancer posed by HPV16 but not by HPV18 was shown in a prospective trial of women with equivocal or mild cervical abnormalities [22]. A likely explanation for increased prevalence of HPV18 in invasive carcinoma but not in high-grade precursor lesions, is an association of HPV18 with a cytopathological effect high in the endocervical canal that is likely to be missed by cytology.

These findings strongly suggest that in particular women who are HPV16 or HPV18 positive should be monitored very closely, even if their smears are cytologically normal.

\section{HPV detection methods}

Because HPV cannot be cultured, all HPV tests currently in use rely on the detection of viral nucleic acids. HPV detection methods can be divided in targetamplification methods and signal-amplification methods. In the following section, technical aspects of the different currently available HPV detection methods are described.

\subsection{Target amplification techniques}

\subsubsection{Consensus primer polymerase chain reaction $(P C R)$}

In most of the PCR-based HPV detection systems, a broad spectrum of HPV types is amplified by consen- 
sus primers, followed by detection with type-specific probes. The consensus primers may be degenerate as in the MY09/11 [23] and CPI/II [24] systems. Alternatively, they may contain mismatches that are accepted under low-stringency PCR conditions as in the GP5+/6+ system [25], they may contain inosine residues at ambiguous base positions such as in the IU/IWDO [26] and SPF [27] primers, or sets of overlapping primers as is the case in the PGMY [28] and Amplicor [29] systems.

\subsubsection{Detection and/or genotyping of consensus PCR products}

For group-specific detection of HPVs without highresolution typing, an enzyme immuno assay (EIA) can be applied conveniently using cocktails of e.g. HR- or LR-HPV probes [30,31].

Typing of PCR products was traditionally done by means of dot blotting or Southern blotting and hybridization with type-specific oligonucleotides. More recently, reverse hybridization techniques were introduced. These methods rely on the hybridization of labelled consensus PCR products to HPV-type specific oligos immobilized on filters. Examples are reverse line blot (RLB) analysis following MY09/11 [23] or GP5 $+/ 6+[25]$ consensus PCR, or a line probe assay (LiPa) following SPF PCR [27]. Detection of the hybridized PCR product is done by a colorimetric reaction $[23,27]$ or by chemiluminescence [25], the latter allowing repeated usage of the same filter [25]. Instead of filters, glass microarrays of HPV type-specific probes can also be used [32]. Recently, a quantitative and high-throughput method was developed [33] based on Luminex suspension array technology. This method relies on detection of consensus primer PCR (GP5+/6+) products with type-specific oligonucleotide probes coupled to fluorescence-labelled polystyrene beads and allows detection of up to 100 different HPV types simultaneously.

Two non-hybridization typing methods following consensus PCR are sequence analysis of the PCR product $[34,35]$ and restriction fragment length polymorphism (RFLP) analysis [36]. RFLP implies the digestion of consensus PCR products with restriction endonucleases, and comparison of the digestion pattern with those of known HPV types. These techniques are useful if unknown types of HPV are present in the specimens, but they have several drawbacks as compared with hybridization methods. For example, RFLP and sequence analysis are not suitable for the detection of infections with multiple HPV types: these will usually give an uninterpretable mix-up of digestion/sequence patterns. In addition, these two methods are less suitable for high-throughput analyses because they are relatively laborious. Finally, RFLP and sequence analysis are less sensitive than hybridization methods because more PCR product is needed to generate a positive signal.

As opposed to typing after consensus PCR, it is also possible to type during the reaction, in real time. Various real-time PCR techniques are available (see also Section 3.2.2), the best known being molecular beacons, the fluorescent 5' exonuclease assay (e.g. Taqman) and fluorescence energy resonance transfer assays (e.g. LightCycler).

At present the only real-time assay that allows consensus HPV-PCR with simultaneous typing is a molecular beacons assay [37]. However, the multiplicity of this technique is limited because the current generation of real-time thermocyclers do not allow for more than six differentially labelled probes. Hence, for typing of multiple HPV infections, reverse hybridization methods are preferred over real-time assays.

\subsubsection{Type-specific PCR}

If one is interested in a particular HPV type, typespecific PCR can be applied (described in [25,38] among others). Care should be taken when designing primers, because they may still react with other types if chosen in well-conserved regions. Confirmation of the specificity of type-specific PCRs, as with consensus primer PCRs, can be done by (regular or reverse) filter hybridization or by EIA, but also in real-time [39-41]. A great advantage of real-time PCR assays is the possibility to quantify the HPV in the specimen. Several studies have shown that the amount of hrHPV present in a cervical smear (the "viral load") as measured by real-time PCR is predictive for the presence or development of high-grade cervical lesions [42-45]; reviewed in [46]. However, to obtain reliable quantification data DNA extraction is usually necessary, thus increasing the work load.

\subsection{4. $m R N A$ amplification}

Several recent studies have shown that hrHPV testing can also be done through detection of the viral $\mathrm{mR}$ NA. The most relevant transcripts to look for are those encoding the viral oncoproteins E6 and E7. It is hypothesized that the presence of viral E6/E7 mRNA in a cervical smear has a better positive predictive value for high-grade cervical lesions than the presence of viral DNA, because the E6/E7 mRNA represents an active 
infection with cell- transforming potential whereas the viral DNA may also be present in clinically irrelevant conditions.

Detection of viral mRNA in cervical smears can be done by reverse-transcriptase (RT-) PCR [47] or by nucleic acid sequence-based amplification (NASBA) [48]. For the latter assay type, a commercially available system was recently developed that detects E6/E7 transcripts from the five most common hrHPV types 16 , 18, 31, 33 and 45 [49-53]. In hospital-based populations, hrHPV mRNA detection showed a better specificity for the detection of high-grade cervical lesions than hrHPV DNA detection [51,52]. However, this method still needs to be validated for population-based screening and triage of women with smears showing minor cellular abnormalities.

An alternative method relying on hrHPV transcription is the detection of HPV-human fusion transcripts that arise from hrHPV genomes integrated into the host cell genome. This method was designated "amplification of papillomavirus oncogene transcripts" (APOT) [54].

Although this method is rather laborious and therefore not suitable for large-scale hrHPV testing, it can give information that is not obtained using other $\mathrm{mR}$ NA amplification techniques. On the one hand, it reveals viral integration, an event that is specific for a real precancerous lesion as opposed to a productive viral infection [55]. On the other hand, it allows the identification of patient-specific fusion transcripts. This may be useful in monitoring of women who have been treated for high-grade lesions, because it will help to determine whether a high-grade lesion occurring after treatment is a recurrence or even a metastasis of the original lesion [56].

\subsection{Signal-amplification techniques}

\subsubsection{Liquid-phase signal amplification techniques}

The best known technique in this category is the commercially available, FDA-approved and clinically validated Hybrid Capture 2 (HC2) method [57]. This method uses a mixture of full-length RNA probes representing hrHPV types $16,18,31,33,35,39,45,51,52$, $56,58,59$, and 68 . Prior to the test, the clinical samples are heat-alkaline-denatured. Hybridization of one or more of the probes to HPV DNA present in the samples is detected by peroxidase-labelled antibodies that recognize the RNA/DNA hybrid, and visualized by chemiluminescence. The analytical sensitivity of this method is $1 \mathrm{pg} / \mathrm{ml}$ of cloned HPV16 DNA, which corresponds to approximately $10^{5}$ HPV16 genome copies. This sensitivity is lower than that of most target-amplification methods. Because a mixture of probes is used, $\mathrm{HC} 2$ is at present not suitable for high-resolution typing. Some cross reactivity of the HC2 probes with HPV types not represented in the probe mix, including non-oncogenic HPVs, has been described [58].

\subsubsection{Morphological signal-amplification techniques}

In addition to the abovementioned methods, hrHPV detection can be performed by DNA in situ hybridization (ISH) to cytological slides $[59,60]$ and histological preparations [61]. This can be achieved by fluorescent detection [61] or colored substrate deposition and bright field microscopy. The relatively small size of the HPV genome $(7.8 \mathrm{~kb})$ and thus of the probe precludes direct detection of hybrids in case of low viral genome copy numbers and therefore some type of signal amplification is generally necessary. A commercially available HPV ISH system uses an indirect biotin-streptavidin method (Ventana Inform HPV) which at present lacks sufficient sensitivity to detect high-grade cervical lesions [60]. Alternatively, tyramide signal amplification (TSA), also known as catalysed reporter deposition (CARD) can be used, both in fluorescent [61,62] and bright field [62] applications. Also for the CARD method, a commercially available system exists (Dako GenPoint). CARD greatly enhances sensitivity, but in general HPV ISH is too laborious to be used in high-throughput HPV testing.

\section{Clinical specimens for HPV detection}

The kind of clinical specimens available will determine the choice of the HPV detection method. Most PCR methods mentioned above can be done with any type of clinical material, including formalin-fixed, paraffin-embedded tissue and Pap-stained archival smears. The recent focus on liquid-based cytology (LBC) for cervical cancer screening has initiated numerous studies evaluating the feasibility of different HPV detection methods on cell suspensions destined for LBC, or cell suspensions remaining after LBC preparation. Some of the pros and cons of different types of clinical specimens are discussed below. 


\subsection{Clinical specimen types suitable for target amplification methods}

\subsubsection{Specimen types for PCR-based methods}

PCR-based HPV DNA detection methods can generally be applied to all kinds of clinical specimens, provided the nucleic acids contained within are not heavily degraded or cross-linked and no putative PCR-inhibiting substances (e.g. organic solvents) are present. Notably, the nucleic acids in formalin-fixed, paraffin-embedded (FFPE) tissue are only amplified in a reliable manner when PCR products are generated that are smaller than $150 \mathrm{bp}$ due to fixative-induced cross links [63]. On the other hand, this type of specimen generally does not contain large amounts of PCRinhibiting substances, as demonstrated by the fact that a relatively crude extract can be used in the PCR without laborious extraction protocols [64].

As opposed to FFPE material, cervical smears collected in LBC media may show excellent preservation of cell morphology and integrity of nucleic acids. However, to achieve this preservation they usually contain relatively high amounts of organic solvents which will inhibit the PCR and need to be removed by careful extraction. An exception are smears collected in Digene Universal Collection Medium (UCM) [65,66], which can be used directly in the PCR after a simple dilution and freeze-thaw protocol (Hesselink et al., submitted).

Pap-stained archival cervical smears can also be used for PCR purposes, but they also need extraction of nucleic acids to remove remnants of dyes. In addition, the nucleic acids in this type of specimen may also be degraded due to fixation artefacts. For all abovementioned samples, careful analysis of the nucleic acid quality is recommended to exclude false negative HPV test results due to DNA degradation and/or PCR inhibition. This can be achieved e.g. by a PCR specific for a human single-copy gene [67].

For most reliable PCR results, it is recommended to standardize the amount of input DNA in the PCR. It is self-evident that this can only be done using purified nucleic acids.

\subsubsection{Specimen types for $m R N A$ amplification methods}

Until recently, hrHPV mRNA testing on cervical smears was hampered by the lack of suitable collection media. The poor RNA quality of samples collected in solutions such as PBS precluded transcription analysis. However, use of LBC is increasing and this is accompanied by the introduction of preservation media that not only safeguard cellular morphology but also the integrity of DNA and mRNA. For example, recent studies have shown that cervical smears collected in PreservCyt LBC medium are of sufficient quality for HPV RT-PCR [68] and NASBA analysis [69].

Alternatively, cervical smears could be collected in a dedicated RNA-preservation buffer [51], but in general these contain chaotropic salts such as guanidinium isothiocyanate and thus destroy cellular morphology.

When hrHPV mRNA is to be detected in cervical biopsy specimens, these should preferably be snapfrozen to guarantee mRNA integrity. However, several groups claim successful mRNA extraction from FFPE material (reviewed in [70]). Provided the use of proper quality controls, this is of course a very interesting source of material, especially for retrospective studies or in situations where snap-freezing is not an option.

\subsection{Clinical specimen types suitable for signal-amplification methods}

The Digene HC2 method is FDA-approved for use on cervical smears and cervical biopsies collected in Digene's Sample Transport Medium (STM). In addition, the FDA-approval of HC2 includes use on cervical smears collected in Cytyc's ThinPrep medium. However, because this medium preserves cell integrity as opposed to STM, ThinPrep smears require an additional pre-treatment to obtain full cell lysis and release of nucleic acids [71]. With some modifications of the standard protocol, HC2 can also be used on the residual material of cervical smears collected in SurePath fluid (i.e. the suspension that remains after the LBC slides have been prepared) [72-74].

An intermediate between the commercially available LBC media that preserve cell morphology but require pre-treatment steps prior to HC2, and Digene's STM that does not require pre-treatment but destroys cell morphology, is Digene Universal Collection Medium (UCM). This medium can be used for sample transport [66], does not require additional steps prior to $\mathrm{HC} 2$ analysis, and because it preserves cell morphology it also allows LBC [65].

\section{Considerations regarding test sensitivity and specificity}

\subsection{Considerations for primary screening and triage}

In general, the analytical sensitivities of HPV tests depend on the test system (target amplification ver- 
sus signal amplification). The sensitivity of the HC2 method is approximately 5,000 copies of the HPV genome per reaction well according to the manufacturer. PCR-based techniques have a much higher analytical sensitivity than $\mathrm{HC} 2$, with a slight variation between the methods (reviewed in [46]). For most PCR assays, less than 100 to $1000 \mathrm{HPV}$ genome equivalents in a reaction tube are sufficient to generate a positive PCR signal and to enable typing.

Despite these high analytical sensitivities, currently available hrHPV tests miss a subset of high-grade CIN lesions [75,76]. This may be due to several factors. For PCR-based methods, modification or loss of primer binding sites due to viral integration or naturally occurring sequence variants may be a problem. For HC2 where whole-genome probes are used this is generally not an issue, but then the lower analytical sensitivity of $\mathrm{HC} 2$ might preclude detection of lesions with low viral loads. The ideal hrHPV test used in primary screening should combine the high analytical sensitivity of PCR assays with the possibility to detect all configurations and variations of the hrHPV genome, including integrated genomes. Because the intact E6/E7 region of the hrHPV genome is necessary for cellular transformation [77] and is invariably retained upon integration [78], a PCR system detecting this region would be the method of choice [79]. It is self-evident that amplification primers should then be chosen in such a way that they cover all known HPV genomic variations.

On the other hand, a very sensitive hrHPV test consequently has a low clinical specificity, and as a primary screening tool it would result in a substantial increase in referrals for colposcopy and repeat smears. In the Dutch screening population, approximately $5.0 \%$ of the women is hrHPV DNA-positive by the GP5+/6+ system [18]. Because even women with hrHPV DNApositive normal cytology have a 210 times greater risk to develop CIN 3 compared to women with hrHPV negative normal cytology [80], all 5.0\% hrHPV DNApositive women would need follow-up. However, only $10 \%$ of them will actually develop CIN3 [81]. This calls for additional stratification of women who have a positive hrHPV DNA test, which can be achieved for example by viral load analysis or HPV mRNA analysis (see Sections 2.1.3 and 2.1.4).

\subsection{Considerations for situations not involving triage}

For several research questions, clinical specificity of the hrHPV test is not an issue as it is in population-based screening and triage. Methods with high analytical sensitivity are especially suitable for studies in which any HPV infection should be detected, irrespective of its clinical relevance. Examples are epidemiological studies, and studies to determine vaccination efficacy. It is self-evident that HPV genotyping is desired in such studies.

\section{Conclusions}

As seen in the previous paragraphs, the aim of the study and the clinical material available will determine the choice of the HPV detection method. In general, consensus PCR followed by reverse hybridization is very sensitive and gives the most extensive typing information for many kinds of clinical specimens, including those containing multiple infections.

In general, it is to be expected that future amplification systems will become faster and more automated.

\section{References}

[1] A.T. Lorincz and R.M. Richart, Human Papillomavirus DNA Testing as an Adjunct to Cytology in Cervical Screening Programs, Arch Pathol Lab Med 127 (2003), 959-968.

[2] J. Cuzick, A. Szarewski, H. Cubie et al., Management of women who test positive for high-risk types of human papillomavirus: the HART study, Lancet 362 (2003), 1871-1876.

[3] A.G. Bais, M. Rebolj, P.J. Snijders et al., Triage using HPVtesting in persistent borderline and mildly dyskaryotic smears: Proposal for new guidelines, Int J Cancer 116 (2005), 122 129.

[4] G.D. Zielinski, A.G. Bais, T.J. Helmerhorst et al., HPV testing and monitoring of women after treatment of CIN 3: review of the literature and meta-analysis, Obstet Gynecol Surv 59 (2004), 543-553.

[5] M. Scheffner, B.A. Werness, J.M. Huibregtse, A.J. Levine and P.M. Howley, The E6 oncoprotein encoded by human papillomavirus types 16 and 18 promotes the degradation of p53, Cell 63 (1990), 1129-1136.

[6] N. Dyson, P.M. Howley, K. Munger and E. Harlow, The human papilloma virus-16 E7 oncoprotein is able to bind to the retinoblastoma gene product, Science 243 (1989), 934-937.

[7] F.X. Bosch, A. Lorincz, N. Munoz, C.J. Meijer and K.V. Shah, The causal relation between human papillomavirus and cervical cancer, J Clin Pathol 55 (2002), 244-265.

[8] N. Munoz, F.X. Bosch, S. de Sanjose et al., Epidemiologic classification of human papillomavirus types associated with cervical cancer, N Engl J Med 348 (2003), 518-527.

[9] E.M. de Villiers, C. Fauquet, T.R. Broker, H.U. Bernard and H. zur Hausen, Classification of papillomaviruses, Virology 324 (2004), 17-27.

[10] B.A. Werness, A.J. Levine and P.M. Howley, Association of human papillomavirus types 16 and 18 E6 proteins with p53, Science 248 (1990), 76-79.

[11] J.R. Gage, C. Meyers and F.O. Wettstein, The E7 proteins of the nononcogenic human papillomavirus type 6b (HPV-6b) and of the oncogenic HPV-16 differ in retinoblastoma protein binding and other properties, J Virol 64 (1990), 723-730. 
[12] Y. Kirii and T. Matsukura, Nucleotide sequence and phylogenetic classification of human papillomavirus type 67, Virus Genes 17 (1998), 117-121.

[13] N. Munoz, F.X. Bosch, X. Castellsague et al., Against which human papillomavirus types shall we vaccinate and screen? The international perspective, Int J Cancer 111 (2004), 278285.

[14] M. Schiffman, M.J. Khan, D. Solomon et al., A study of the impact of adding HPV types to cervical cancer screening and triage tests, J Natl Cancer Inst 97 (2005), 147-150.

[15] D.A. Galloway, Papillomavirus vaccines in clinical trials, Lancet Infect Dis 3 (2003), 469-475.

[16] A.H. Beskow, M. Moberg and U.B. Gyllensten, HLA class II allele control of HPV load in carcinoma in situ of the cervix uteri, Int J Cancer 117 (2005), 510-514.

[17] A.H. Beskow and U.B. Gyllensten, Host genetic control of HPV 16 titer in carcinoma in situ of the cervix uteri, Int $J$ Cancer 101 (2002), 526-531.

[18] N.W.J. Bulkmans, L. Rozendaal, P.J. Snijders et al., POBASCAM, a population-based randomised controlled trial for implementation of high-risk HPV testing in cervical screening; Design, methods and baseline data of 44,102 women, Int $J$ Cancer 110 (2003), 94-101.

[19] N.W. Bulkmans, M.C. Bleeker, J. Berkhof et al., Prevalence of types 16 and 33 is increased in high-risk human papillomavirus positive women with cervical intraepithelial neoplasia grade 2 or worse, Int J Cancer 117 (2005), 177-181.

[20] S. Bulk, J. Berkhof, N.W. Bulkmans et al., Preferential risk of HPV16 for squamous cell carcinoma and of HPV18 for adenocarcinoma of the cervix compared to women with normal cytology in The Netherlands, Br J Cancer 94 (2006), 171-175.

[21] M.J. Khan, P.E. Castle, A.T. Lorincz et al., The elevated 10year risk of cervical precancer and cancer in women with human papillomavirus (HPV) type 16 or 18 and the possible utility of type-specific HPV testing in clinical practice, $J$ Natl Cancer Inst 97 (2005), 1072-1079.

[22] P.E. Castle, D. Solomon, M. Schiffman and C.M. Wheeler, Human papillomavirus type 16 infections and 2-year absolute risk of cervical precancer in women with equivocal or mild cytologic abnormalities, J Natl Cancer Inst 97 (2005), 10661071.

[23] P.E. Gravitt, C.L. Peyton, R.J. Apple and C.M. Wheeler, Genotyping of 27 human papillomavirus types by using L1 consensus PCR products by a single-hybridization, reverse line blot detection method, J Clin Microbiol 36 (1998), 3020-3027.

[24] L.M. Tieben, J. ter Schegget, R.P. Minnaar et al., Detection of cutaneous and genital HPV types in clinical samples by PCR using consensus primers, J Virol Methods 42 (1993), 265-279.

[25] A.J. van den Brule, R. Pol, N. Fransen-Daalmeijer et al., GP5+/6+ PCR followed by reverse line blot analysis enables rapid and high-throughput identification of human papillomavirus genotypes, J Clin Microbiol 40 (2002), 779-787.

[26] L. Gregoire, M. Arella, J. Campione-Piccardo and W.D. Lancaster, Amplification of human papillomavirus DNA sequences by using conserved primers, J Clin Microbiol 27 (1989), 2660-2665.

[27] B. Kleter, L.J. van Doorn, L. Schrauwen et al., Development and clinical evaluation of a highly sensitive PCR-reverse hybridization line probe assay for detection and identification of anogenital human papillomavirus, J Clin Microbiol 37 (1999), 2508-2517.

[28] P.E. Gravitt, C.L. Peyton, T.Q. Alessi et al., Improved amplification of genital human papillomaviruses, J Clin Microbiol 38 (2000), 357-361
[29] J. Monsonego, J.M. Bohbot, G. Pollini et al., Performance of the Roche AMPLICOR human papillomavirus (HPV) test in prediction of cervical intraepithelial neoplasia (CIN) in women with abnormal PAP smear, Gynecol Oncol 99 (2005), 160168.

[30] M.V. Jacobs, P.J. Snijders, A.J. van den Brule et al., A general primer GP5+/GP6(+)-mediated PCR-enzyme immunoassay method for rapid detection of 14 high-risk and 6 low-risk human papillomavirus genotypes in cervical scrapings, J Clin Microbiol 35 (1997), 791-795.

[31] B. Kleter, L.J. van Doorn, J. ter Schegget et al., Novel shortfragment PCR assay for highly sensitive broad-spectrum detection of anogenital human papillomaviruses, Am J Pathol 153 (1998), 1731-1739.

[32] N.H. Cho, H.J. An, J.K. Jeong et al., Genotyping of 22 human papillomavirus types by DNA chip in Korean women: comparison with cytologic diagnosis, Am J Obstet Gynecol 188 (2003), 56-62.

[33] M. Schmitt, I.G. Bravo, P.J. Snijders et al., Bead-based multiplex genotyping of human papillomaviruses, J Clin Microbiol 44 (2006), 504-512.

[34] O. Forslund, A. Antonsson, P. Nordin, B. Stenquist and B.G. Hansson, A broad range of human papillomavirus types detected with a general PCR method suitable for analysis of cutaneous tumours and normal skin, J Gen Virol 80(Pt 9) (1999), 2437-2443.

[35] V. Shamanin, M. Glover, C. Rausch et al., Specific types of human papillomavirus found in benign proliferations and carcinomas of the skin in immunosuppressed patients, Cancer Res 54 (1994), 4610-4613.

[36] O. Lungu, T.C. Wright Jr. and S. Silverstein, Typing of human papillomaviruses by polymerase chain reaction amplification with L1 consensus primers and RFLP analysis, Mol Cell Probes 6 (1992), 145-152.

[37] K. Szuhai, E. Sandhaus, S.M. Kolkman-Uljee et al., A novel strategy for HPV detection and genotyping with SybrGreen and Molecular Beacon PCR, Am J Pathol 159 (2001), 16511660.

[38] J.M. Walboomers, M.V. Jacobs, M.M. Manos et al., Human papillomavirus is a necessary cause of invasive cervical cancer worldwide, J Pathol 189 (1999), 12-19.

[39] A. Josefsson, K. Livak and U. Gyllensten, Detection and quantitation of human papillomavirus by using the fluorescent 5 , exonuclease assay, J Clin Microbiol 37 (1999), 490-496.

[40] A.T. Hesselink, A.J. van den Brule, Z.M. Groothuismink et al., Comparison of three different PCR methods for quantifying human papillomavirus type 16 DNA in cervical scrape specimens, J Clin Microbiol 43 (2005), 4868-4871.

[41] M. Moberg, I. Gustavsson and U. Gyllensten, Real-time PCRbased system for simultaneous quantification of human papillomavirus types associated with high risk of cervical cancer, $J$ Clin Microbiol 41 (2003), 3221-3228.

[42] A.M. Josefsson, P.K. Magnusson, N. Ylitalo et al., Viral load of human papilloma virus 16 as a determinant for development of cervical carcinoma in situ: a nested case-control study, Lancet 355 (2000), 2189-2193.

[43] N. Ylitalo, P. Sorensen, A.M. Josefsson et al., Consistent high viral load of human papillomavirus 16 and risk of cervical carcinoma in situ: a nested case-control study, Lancet $\mathbf{3 5 5}$ (2000), 2194-2198.

[44] M. van Duin, P.J. Snijders, H.F. Schrijnemakers et al., Human papillomavirus 16 load in normal and abnormal cervical scrapes: an indicator of CIN II/III and viral clearance, Int $J$ Cancer 98 (2002), 590-595. 
[45] P.J. Snijders, C.J. Hogewoning, A.T. Hesselink et al., Determination of viral load thresholds in cervical scrapings to rule out CIN 3 in HPV 16, 18, 31 and 33-positive women with normal cytology, Int J Cancer (2006).

[46] P.J. Snijders, A.J. van den Brule and C.J. Meijer, The clinical relevance of human papillomavirus testing: relationship between analytical and clinical sensitivity, J Pathol 201 (2003), $1-6$.

[47] K. Sotlar, A. Stubner, D. Diemer et al., Detection of highrisk human papillomavirus E6 and E7 oncogene transcripts in cervical scrapes by nested RT-polymerase chain reaction, $J$ Med Virol 74 (2004), 107-116.

[48] H.L. Smits, B. van Gemen, R. Schukkink et al., Application of the NASBA nucleic acid amplification method for the detection of human papillomavirus type 16 E6-E7 transcripts, $J$ Virol Methods 54 (1995), 75-81.

[49] K.S. Cuschieri, M.J. Whitley and H.A. Cubie, Human papillomavirus type specific DNA and RNA persistence-implications for cervical disease progression and monitoring, $\mathrm{J}$ Med Virol 73 (2004), 65-70.

[50] I. Kraus, T. Molden, L.E. Erno et al., Human papillomavirus oncogenic expression in the dysplastic portio; an investigation of biopsies from 190 cervical cones, Br J Cancer 90 (2004), 1407-1413.

[51] T. Molden, I. Kraus, F. Karlsen et al., Comparison of human papillomavirus messenger RNA and DNA detection: a crosssectional study of 4,136 women $>30$ years of age with a 2year follow-up of high-grade squamous intraepithelial lesion, Cancer Epidemiol Biomarkers Prev 14 (2005), 367-372.

[52] T. Molden, J.F. Nygard, I. Kraus et al., Predicting CIN2+ when detecting HPV mRNA and DNA by PreTect HPVproofer and consensus PCR: A 2-year follow-up of women with ASCUS or LSIL Pap smear, Int J Cancer 114 (2005), 973-976.

[53] T. Molden, I. Kraus, F. Karlsen, H. Skomedal and B. Hagmar, Human papillomavirus E6/E7 mRNA expression in women younger than 30 years of age, Gynecol Oncol 100 (2005), 95-100.

[54] R. Klaes, S.M. Woerner, R. Ridder et al., Detection of highrisk cervical intraepithelial neoplasia and cervical cancer by amplification of transcripts derived from integrated papillomavirus oncogenes, Cancer Res 59 (1999), 6132-6136.

[55] N. Wentzensen, R. Ridder, R. Klaes et al., Characterization of viral-cellular fusion transcripts in a large series of HPV 16 and 18 positive anogenital lesions, Oncogene 21 (2002), 419-426.

[56] S. Vinokurova, N. Wentzensen, J. Einenkel et al., Clonal history of papillomavirus-induced dysplasia in the female lower genital tract, J Natl Cancer Inst 97 (2005), 1816-1821.

[57] C. Clavel, M. Masure, I. Putaud et al., Hybrid capture II, a new sensitive test for human papillomavirus detection. Comparison with hybrid capture I and PCR results in cervical lesions, $J$ Clin Pathol 51 (1998), 737-740.

[58] P.E. Castle, M. Schiffman, R.D. Burk et al., Restricted crossreactivity of hybrid capture 2 with nononcogenic human papillomavirus types, Cancer Epidemiol Biomarkers Prev 11 (2002), 1394-1399.

[59] M.N. Qureshi, R.D. Rudelli, R.R. Tubbs, C.V. Biscotti and L.J. Layfield, Role of HPV DNA testing in predicting cervical intraepithelial lesions: comparison of HC HPV and ISH HPV, Diagn Cytopathol 29 (2003), 149-155.

[60] A.T. Hesselink, A.J. van den Brule, A.A. Brink et al., Comparison of hybrid capture 2 with in situ hybridization for the detection of high-risk human papillomavirus in liquid-based cervical samples, Cancer 102 (2004), 11-18.
[61] A.H. Hopman, F. Smedts, W. Dignef et al., Transition of high-grade cervical intraepithelial neoplasia to micro-invasive carcinoma is characterized by integration of HPV 16/18 and numerical chromosome abnormalities, J Pathol 202 (2004), 23-33.

[62] H.M. Kerstens, P.J. Poddighe and A.G. Hanselaar, A novel in situ hybridization signal amplification method based on the deposition of biotinylated tyramine, J Histochem Cytochem 43 (1995), 347-352.

63] C.C. Impraim, R.K. Saiki, H.A. Erlich and R.L. Teplitz, Analysis of DNA extracted from formalin-fixed, paraffinembedded tissues by enzymatic amplification and hybridization with sequence-specific oligonucleotides, Biochem Biophys Res Commun 142 (1987), 710-716.

[64] R.J. Slebos, L. Boerrigter, S.G. Evers et al., A rapid and simple procedure for the routine detection of ras point mutations in formalin-fixed, paraffin-embedded tissues, Diagn Mol Pathol 1 (1992), 136-141.

[65] Mattosinho de Castro Ferraz Mda, S.M. Nicolau, J.N. Stavale et al., Cervical biopsy-based comparison of a new liquid-based thin-layer preparation with conventional Pap smears, Diagn Cytopathol 30 (2004), 220-226.

[66] N.S. Taha, J. Focchi, J.C. Ribalta et al., Universal Collection Medium (UCM) ${ }^{\circledR}$ is as suitable as the Standard Transport Medium (STM) ${ }^{\circledR}$ for Hybrid Capture II ${ }^{\circledR}(\mathrm{HC}-2)$ assay, J Clin Virol 36 (2006), 32-35.

[67] A.M. de Roda Husman, P.J. Snijders, H.V. Stel et al., Processing of long-stored archival cervical smears for human papillomavirus detection by the polymerase chain reaction, $\mathrm{Br} \mathrm{J}$ Cancer 72 (1995), 412-417.

[68] T.A. Tarkowski, M.S. Rajeevan, D.R. Lee and E.R. Unger, Improved detection of viral RNA isolated from liquid-based cytology samples, Mol Diagn 6 (2001), 125-130.

[69] A.K. Lie, B. Risberg, B. Borge et al., DNA- versus RNAbased methods for human papillomavirus detection in cervical neoplasia, Gynecol Oncol 97 (2005), 908-915.

[70] F. Lewis, N.J. Maughan, V. Smith, K. Hillan and P. Quirke, Unlocking the archive - gene expression in paraffin-embedded tissue, J Pathol 195 (2001), 66-71.

[71] Digene Hybrid Capture 2 high-risk HPV DNA test - part 3 (Labelling). http://www.fda.gov/cdrh/pdf/P890064s009c.pdf, 2006.

[72] M.N. Qureshi, D. Bolick, P.J. Ringer, F.L. Spagler and G. Zimmerman, HPV testing in liquid cytology specimens: comparison of analytic sensitivty and specificity for in situ hybridization and chemiluminescent nucleic acid testing, Acta Cytol 49 (2005), 120-126.

[73] P. Vassilakos, P. Petignat, M. Boulvain and A. Campana, Primary screening for cervical cancer precursors by the combined use of liquid-based cytology, computer-assisted cytology and HPV DNA testing, Br J Cancer 86 (2002), 382-388.

[74] S. Davis-Devine, S.J. Day and G.G. Freund, Test performance comparison of inform HPV and hybrid capture 2 high-risk HPV DNA tests using the SurePath liquid-based Pap test as the collection method, Am J Clin Pathol 124 (2005), 24-30.

[75] G.D. Zielinski, L. Rozendaal, F.J. Voorhorst et al., HPV testing can reduce the number of follow-up visits in women treated for cervical intraepithelial neoplasia grade 3, Gynecol Oncol 91 (2003), 67-73.

[76] M.A. Nobbenhuis, C.J. Meijer, A.J. van den Brule et al., Addition of high-risk HPV testing improves the current guidelines on follow-up after treatment for cervical intraepithelial neoplasia, Br J Cancer 84 (2001), 796-801. 
[77] P. Hawley-Nelson, K.H. Vousden, N.L. Hubbert, D.R. Lowy and J.T. Schiller, HPV16 E6 and E7 proteins cooperate to immortalize human foreskin keratinocytes, EMBO J 8 (1989), 3905-3910.

[78] K.B. Choo, C.C. Pan and S.H. Han, Integration of human papillomavirus type 16 into cellular DNA of cervical carcinoma: preferential deletion of the $\mathrm{E} 2$ gene and invariable retention of the long control region and the E6/E7 open reading frames, Virology 161 (1987), 259-261.

[79] A.T. Hesselink, M. Lettink, J. Berkhof, C.J. Meijer and P.J. Snijders, Comparison of a novel consensus high-risk HPV E7 PCR with GP5+/6+ PCR on cervical scrapings. 2006. 6th International Multidisciplinary Congress on Human Papillo- mavirus Infection and Global Prevention of Cervical Cancer EUROGIN. Monsonego, J. 23-4-2006.

[80] L. Rozendaal, J. Westerga, J.C. van der Linden et al., PCR based high risk HPV testing is superior to neural network based screening for predicting incident CIN III in women with normal cytology and borderline changes, J Clin Pathol 53 (2000), 606-611.

[81] L. Rozendaal, J.M. Walboomers, J.C. van der Linden et al., PCR-based high-risk HPV test in cervical cancer screening gives objective risk assessment of women with cytomorphologically normal cervical smears, Int J Cancer 68 (1996), 766-769. 


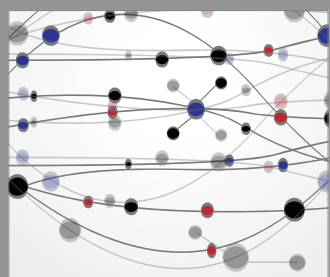

The Scientific World Journal
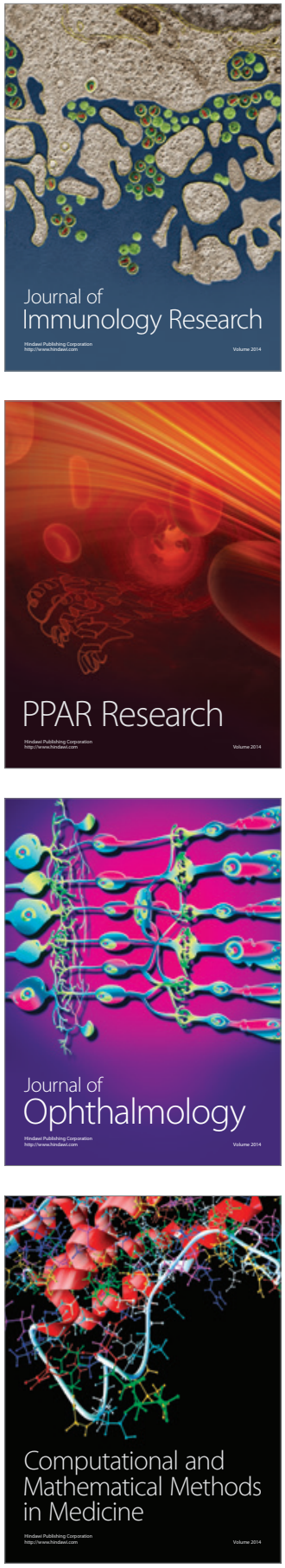

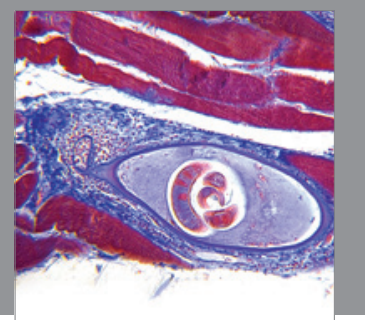

Gastroenterology

Research and Practice
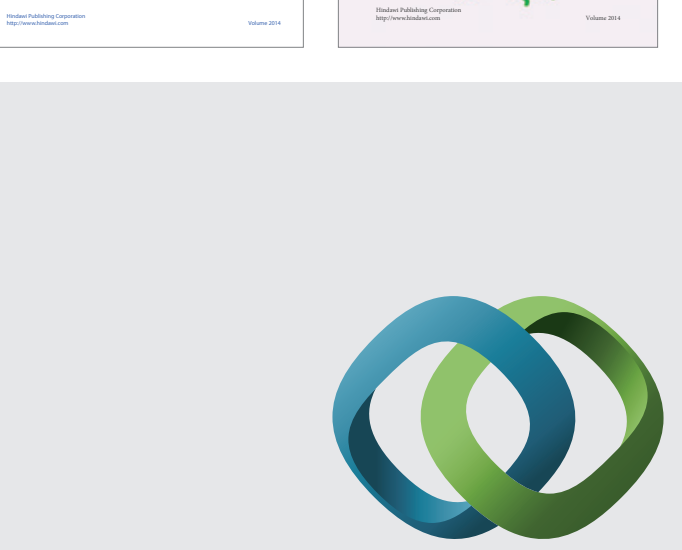

\section{Hindawi}

Submit your manuscripts at

http://www.hindawi.com
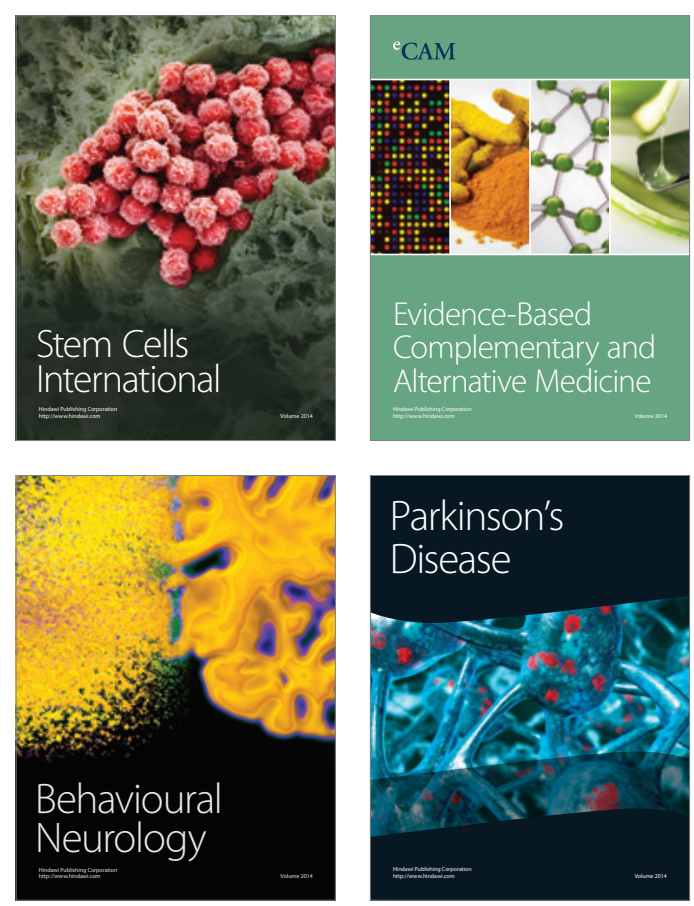

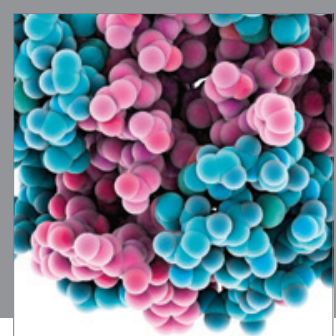

Journal of
Diabetes Research

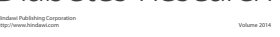

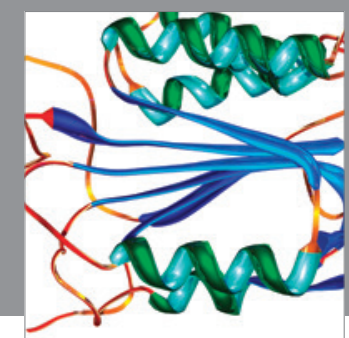

Disease Markers
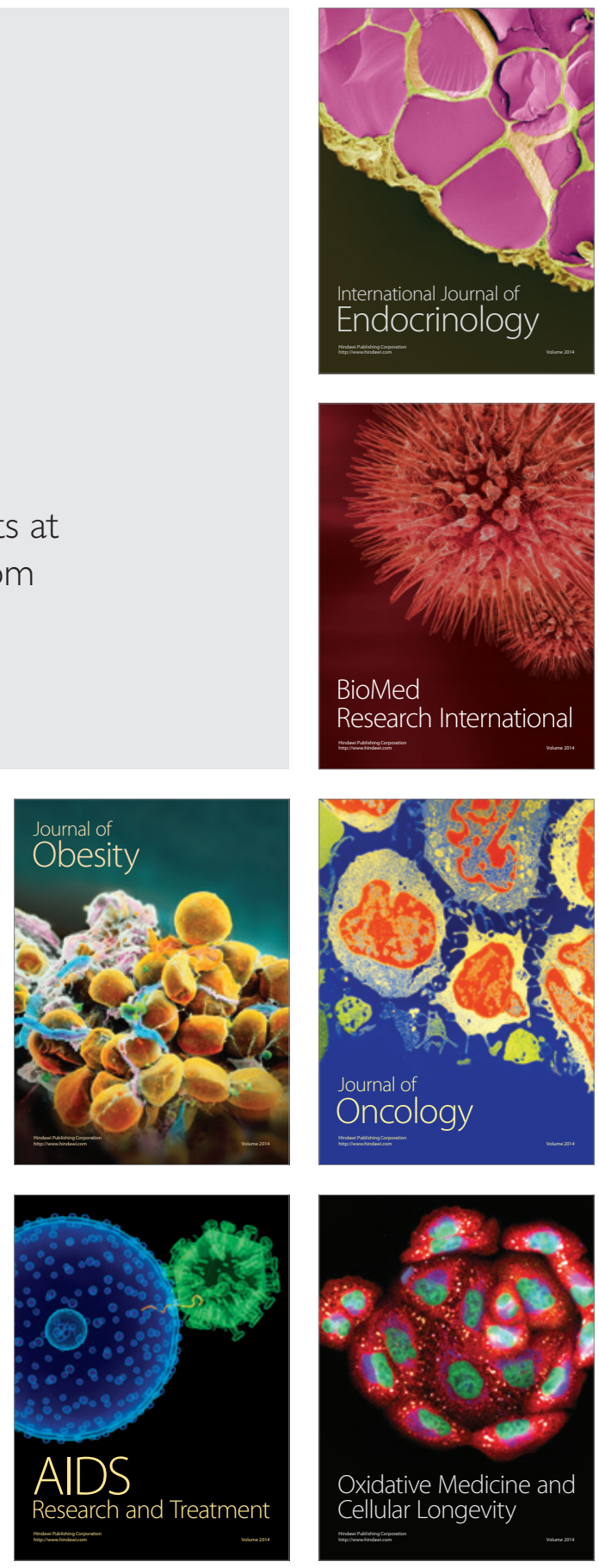